\title{
Erratum to: Protective Effects of Hesperidin Against Amyloid- $\beta$ (Aß) Induced Neurotoxicity Through the Voltage Dependent Anion Channel 1 (VDAC1)-Mediated Mitochondrial Apoptotic Pathway in PC12 Cells
}

\author{
Dong-Mei Wang $\cdot$ San-Qiang $\mathbf{L i} \cdot$ Xiao-Ying Zhu $\cdot$ \\ Yong Wang $\cdot$ Wen-Lan Wu $\cdot$ Xiao-Juan Zhang
}

Published online: 28 April 2013

(C) Springer Science+Business Media New York 2013

\section{Erratum to: Neurochem Res (2013) 38:1034-1044}

DOI 10.1007/s11064-013-1013-4

The following errors were inadvertently overlooked in the original publication and it has been corrected with this erratum.

1. In Abstract Section, "Further study indicated that hesperidin can increase the level of VDAC1 phosphorylation through enhancing the activity of the glycogen synthase kinase- $3 \beta$ and decrease the level of hexokinaseI in mitochondrial, resulting in mitochondrial release of cytochrome c." should be replaced with "Further study indicated that hesperidin can decrease the level of VDAC1 phosphorylation through inhibiting the activity of the glycogen synthase kinase- $3 \beta$ and increase the level of hexokinaseI in mitochondria, preventing release of cytochrome c from mitochondria."

2. In Result Section (p. 1039), "Hesperidin enhances VDAC1 phosphorylation and promotes the release of HXKI from mitochondria in A $\beta 25-35$-treated PC12 cells" should be replaced with "Hesperidin inhibits VDAC1 phosphorylation and prevents the release of HXKI from mitochondria in A $\beta 25-35$-treated PC12 cells."

The online version of the original article can be found under doi:10.1007/s11064-013-1013-4.

D.-M. Wang $(\bowtie) \cdot$ X.-Y. Zhu · Y. Wang · W.-L. Wu •

X.-J. Zhang

Department of Pathogen Biology, Medical College,

Henan University of Science and Technology,

Building 6, Anhui, Jianxi District, Luoyang 471003,

People's Republic of China

e-mail: wdmzgadyx@163.com

S.-Q. Li

Department of Biochemistry and Molecular Biology, Medical

College, Henan University of Science and Technology,

Luoyang, China 